\title{
Automated High Precision Strain Measurement Using Nanobeam Diffraction Coupled with Precession
}


K. Weiss ${ }^{1}$

1. AppFive LLC, Tempe, Arizona, USA.

2. Texas Instruments, Dallas, Texas, USA

3. NanoTEM, Scottsdale, Arizona, USA

${ }^{4 .}$ NanoMEGAS SPRL, Brussels, Belgium

Measurement of strain with high spatial resolution and high precision in semiconductor devices is critical to monitor the designed and unintended strain distributions. For this purpose, spot diffraction patterns acquired using nanobeam illumination in the Transmission Electron Microscope (TEM) have been used. Such patterns can be acquired at high spatial resolution compared to other TEM strain measurement techniques, and the experiment is relatively straightforward to perform on most modern microscopes [1].

Previous studies using nanobeam diffraction have used the measurement of shifts in individual diffraction spots to measure strain [1]. It should be possible to improve precision by matching the entire strained diffraction pattern to a reference pattern, since more data is being utilized. The presence of strong dynamical effects in electron diffraction makes such a scheme difficult since spot intensity distributions are strongly dependent on local specimen thickness, and this causes significant variation in diffraction patterns from different areas. This particular challenge can be overcome by combining nanobeam diffraction with beam precession [2]. With precession the incident beam is tilted and rotated at a high frequency (typically about $100 \mathrm{~Hz}$ ), so the dynamical effects are effectively averaged out. Precession also enables the collection of higher-order reflections, which are more sensitive than lowerorder reflections to small changes in lattice parameters.

In the present work, strain measurements from nanobeam diffraction combined with precession diffraction are presented. Diffraction patterns were obtained with a $200 \mathrm{kV}$ Zeiss Libra L200 TEM equipped with a field emission gun (FEG), operated in the Scanning TEM (STEM) mode. A NanoMEGAS DigiSTAR unit was used to produce precession and descanning of the electron beam. Strain is derived by numerically distorting the reference pattern so that it matches each of the diffraction patterns from the strained region. The processing of diffraction patterns to obtain strain coefficients is relatively simple, since matching entire images means one does not need to identify the coordinates of individual spots.

Two specimens are measured. The first is a cross section of a blanket SiGe layer grown on a singlecrystal Si substrate, prepared using focused ion beam milling. Strain measurements are taken along line profiles parallel and perpendicular to the $\mathrm{Si} / \mathrm{SiGe}$ interface, as shown in Figure 1. For each profile, strains in directions parallel and perpendicular to the boundary are plotted. Strain is also measured for a AlGaN/GaN heterostructure in a High Electron Mobility Transistor (HEMT), where knowledge of the strain distribution is crucial in understanding the reliability of such devices [3]. In this case diffraction patterns are acquired over a 100 by 200 grid of points with a step size of $6 \mathrm{~nm}$ spanning the region shown in Figure 2, where the STEM reference image of the gate structure is on the left hand side. Color coded strain images for strain in directions parallel and perpendicular to the AlGaN/GaN interface are 
also displayed in Figure 2. Calculating the strain values in these images takes a only a few minutes on a modern personal computer. For both specimens the reference diffraction pattern is obtained in areas well away from the boundary regions where strains are expected.

An average normal strain $\varepsilon_{y y}$ of $1.22 \%$ strain was observed in the SiGe layer. The strain parallel to the interface $\varepsilon_{\mathrm{xx}}$ was close to zero, indicating that the $\mathrm{Si} / \mathrm{SiGe}$ interface is coherent. Calculating the variation in strain across uniform regions of the sample indicates that the precision of strain measurements is $0.02 \%$ in this case. Strain maps from the AlGaN/GaN HEMT show an average of $1 \%$ compressive strain perpendicular to the $\mathrm{AlGaN} / \mathrm{GaN}$ interface in the $\mathrm{AlGaN}$ layer. The strain map also shows tensile strain perpendicular to the interface concentrated close to the corners of the gate-dielectric interface.

References:

[1] D Cooper et al., Journal of Physics: Conference Series 326012025 (2011).

[2] R Vincent, PA Midgley, Ultramicroscopy 53 (1994) p. 271.

[3] U Chowdhury, et al., IEEE Electron Device Letters, 29 (2008), 1098-1100.


Figure 1. STEM reference image of Si-SiGe layer (left panel) and strain profiles (right panel).
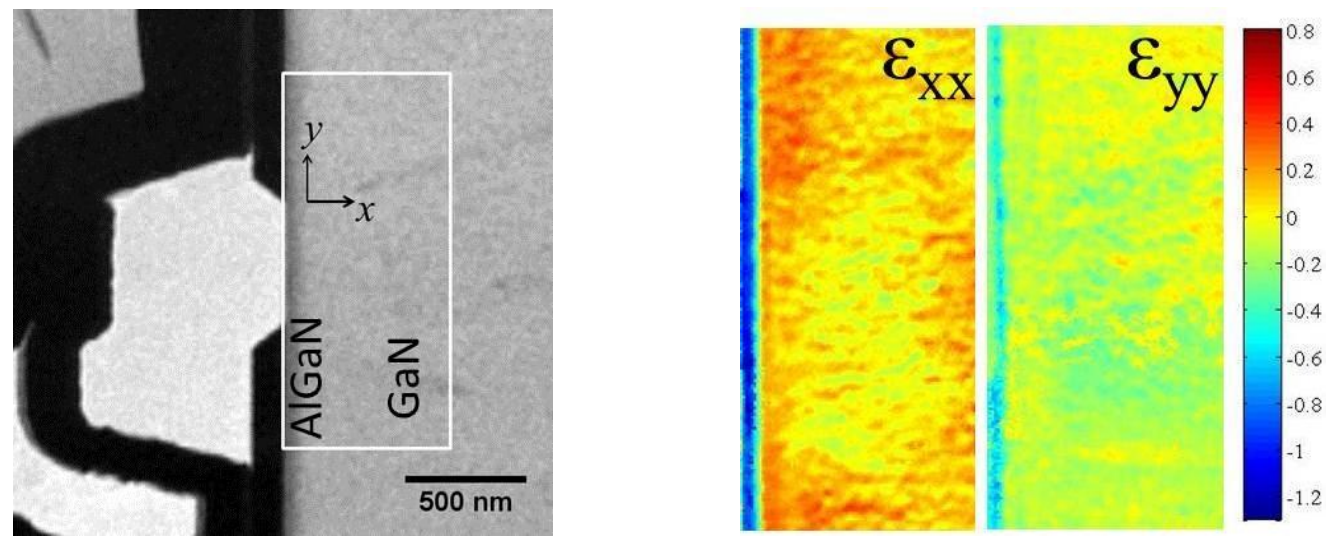

Figure 2. STEM reference image of a GaN HEMT (left panel) and strain images (center and right panels). The white square in the reference image encloses the region where strain is calculated. Color represents percentage strain. $y$ direction is parallel and $x$ direction is perpendicular to the interface. Positive values in the color scale correspond to tensile strain and negative values compressive strain. Regions that do not correlate well with the reference image are masked in white. 